\title{
Existence and Global Uniform Asymptotic Stability of Pseudo Almost Periodic Solutions for Cohen-Grossberg Neural Networks with Discrete and Distributed Delays
}

\author{
Hongying Zhu' ${ }^{1}$ and Chunhua Feng ${ }^{2}$ \\ ${ }^{1}$ School of Information and Statistics, Guangxi University of Finance and Economics, \\ Nanning 530003, China \\ ${ }^{2}$ School of Mathematics Science, Guangxi Normal University, Guiling 541004, China \\ Correspondence should be addressed to Hongying Zhu; zhy71118@163.com
}

Received 11 March 2014; Accepted 28 July 2014; Published 18 August 2014

Academic Editor: Zhichun Yang

Copyright (C) 2014 H. Zhu and C. Feng. This is an open access article distributed under the Creative Commons Attribution License, which permits unrestricted use, distribution, and reproduction in any medium, provided the original work is properly cited.

This paper studies the existence and uniform asymptotic stability of pseudo almost periodic solutions to Cohen-Grossberg neural networks (CGNNs) with discrete and distributed delays by applying Schauder fixed point theorem and constructing a suitable Lyapunov functional. An example is given to show the effectiveness of the main results.

\section{Introduction}

Since the model of Cohen-Grossberg neural networks (CGNNs) was first proposed and studied by Cohen and Grossberg [1], it has been widely investigated because of the theoretical interest as well as the application considerations such as optimization, pattern recognition, automatic control, image processing, and associative memories. In recent years, there are many important results on dynamic behaviors of CGNNs. For instance, many sufficient conditions have been successively obtained to ensure the existence and stability of equilibrium point of CGNNs [1-10]. Some attractivity and asymptotic stability results have also been published $[3,11-14]$. Many authors specially devote themselves to study the existence and global exponential stability of periodic or almost periodic solution to CGNNs [15-30]; for the other dynamic properties, see also the literatures [31,32]. However, to the best of our knowledge, few authors have discussed the existence and the global uniform asymptotic stability of pseudo almost periodic solutions to CGNNs.

In this paper, we discuss the existence and the global uniform asymptotic stability of pseudo almost periodic solutions to the following CGNNs:

$$
\begin{aligned}
x_{i}^{\prime}(t)= & -a_{i}\left(x_{i}(t)\right) \\
& \times\left[b_{i}\left(x_{i}(t)\right)-\sum_{j=1}^{m} c_{i j}(t) f_{j}\left(x_{j}(t)\right)\right. \\
& \quad-\sum_{j=1}^{m} d_{i j}(t) g_{j}\left(x_{j}\left(t-\tau_{i j}(t)\right)\right), \\
& \left.\quad-\sum_{j=1}^{m} p_{i j}(t) \int_{-\infty}^{t} G_{i j}(t-s) h_{j}\left(x_{j}(s)\right) d s-I_{i}(t)\right], \\
x_{i}(t)= & \Phi_{i}(t), \quad t \geq 0,
\end{aligned}
$$

where $c_{i j}(t), d_{i j}(t), p_{i j}(t), I_{i}(t), \Phi_{i}(t) \in C(R, R), \tau_{i j}(t) \in$ $C\left(R, R^{+}\right)$are pseudo almost periodic functions.

The organization of this paper is as follows. In Section 2, some basic definitions, marks, and lemmas are given. In Section 3, some results are given to ascertain the existence of pseudo almost periodic solution to the system (1) by 
applying Schauder fixed point theorem. In Section 4, the global uniform asymptotic stability of pseudo almost periodic solutions to the system (1) is obtained. In Section 5, an example is provided to demonstrate the effectiveness of the main results. In Section 6, the final conclusions are drawn.

\section{Preliminaries}

In this section, some basic definitions, lemmas, and assumptions are introduced.

Definition 1 (see [33]). $f(t) \in B C(R, R)$ is said to be Bohr almost periodic if, for all $\epsilon>0$, set

$$
T(f, \epsilon)=\{|f(t+\tau)-f(t)|<\epsilon, \forall t \in R\}
$$

is relatively dense. Namely, for any $\epsilon>0$ there exists a number $l=l(\epsilon)>0$ such that every interval $[a, a+l]$ contains at least one point of $\tau=\tau(\epsilon)$ such that $|f(t+\tau)-f(t)|<\epsilon$ for every $t \in R$. The collection of those functions is denoted by $A P\left(R, R^{m}\right)$. Define the class of functions $P A P_{0}\left(R, R^{m}\right)$ as follows:

$$
\begin{aligned}
\operatorname{PAP}_{0}\left(R, R^{m}\right)=\{f & \in B C\left(R, R^{m}\right) \mid \\
& \left.\lim _{T \rightarrow+\infty} \frac{1}{2 T} \int_{-T}^{T}\|f(t)\| d t=0\right\} .
\end{aligned}
$$

Definition 2 (see [34]). A function $f \in B C\left(R, R^{m}\right)$ is called pseudo almost periodic if it can be expressed as

$$
f=f_{1}+f_{0},
$$

where $f_{1} \in A P\left(R, R^{m}\right)$ and $f_{0} \in P A P_{0}\left(R, R^{m}\right)$. The collection of such functions will be denoted by $P A P\left(R, R^{m}\right)$.

Remark 3. From the definitions above, we have $A P\left(R, R^{m}\right) \subset$ $\operatorname{PAP}\left(R, R^{m}\right)$.

Lemma 4 (see [3]). $P A P\left(R, R^{m}\right)$ is a Banach space with the norm $|\phi|=\sup _{t \in R}|\phi(t)|$.

Lemma 5 (see [19]). If $f(t, u) \in C\left(R \times D, R^{m}\right)$, where $D$ is an open set in $R^{m}$ or $D=R^{m}, C\left(R \times D, R^{m}\right)$ denote continuous function class. Suppose $f \in P A P(R \times D)$ satisfies the Lipschitz condition

$$
|f(t, u)-f(t, v)| \leq L|u-v|, \quad \forall t \in R, u, v \in D ;
$$

if $\phi(t) \in P A P(R)$, then the composite function $f(t, \phi(t)) \in$ $P A P(R)$. Suppose $f: R \times C \rightarrow R^{m}$; then the equation

$$
x^{\prime}(t)=f\left(t, x_{t}\right)
$$

is called lagging-type almost periodic differential equation. The following system (7) is defined as the product systems of (6):

$$
x^{\prime}(t)=f\left(t, x_{t}(t)\right), \quad y^{\prime}(t)=f\left(t, y_{t}(t)\right) .
$$

Lemma 6. Suppose $\phi(t) \in P A P\left(R, R^{m}\right)$; then $\phi(t-\tau) \in$ $\operatorname{PAP}\left(R, R^{m}\right)$ for all $\tau \in R$.
Proof. From Definition 2 of the PAP, we have $\phi=\phi_{1}+\phi_{0}$, where $\phi_{1} \in A P\left(R, R^{m}\right)$ and $\phi_{0} \in P A P_{0}\left(R, R^{m}\right)$. Clearly $\phi(t-$ $\tau)=\phi_{1}(t-\tau)+\phi_{0}(t-\tau)$; it is easy to $\operatorname{know} \phi_{1}(t-\tau) \in A P\left(R, R^{m}\right)$ and

$$
\begin{aligned}
0 & \leq \frac{1}{2 T} \int_{-T}^{T}\left|\phi_{0}(t-\tau)\right| d t=\frac{1}{2 T} \int_{-(T+\tau)}^{T-\tau}\left|\phi_{0}(t)\right| d t \\
& \leq \frac{T+\tau}{T} \cdot \frac{1}{2(T+\tau)} \int_{-(T+\tau)}^{T+\tau}\left|\phi_{0}(t)\right| d t .
\end{aligned}
$$

This indicates that $\phi_{0}(t-\tau) \in P A P_{0}\left(R, R^{m}\right)$. So $\phi(t-\tau) \epsilon$ $\operatorname{PAP}\left(R, R^{m}\right)$.

Definition 7. Assume that $x^{*}(t)$ is a pseudo almost periodic solution of system (1). By a translation transformation $y(t)=$ $x(t)-x^{*}(t)$, system (1) is transformed into a new system. If the zero solution of new system is globally uniformly asymptotically stable, then the pseudo almost periodic solution of system (1) is said to be globally uniformly asymptotically stable. As for the uniform asymptotical stability, see [35].

Lemma 8 (see [33]). There is a continuous functional $V(t, \varphi, \psi)$ for $t \geq 0, \varphi, \psi \in C_{H}, C_{H}=\{\varphi: \varphi \in C,|\varphi|<H\}$, $|\varphi|=\sup _{\theta \in[-r, 0]}|\varphi(\theta)|$ such that

$$
\begin{aligned}
& \left(H^{\prime} 2.1\right) u(|\varphi-\psi|) \leq V(t, \varphi, \psi) \leq v(|\varphi-\psi|) ; \\
& \left(H^{\prime} 2.2\right)\left|V\left(t, \varphi_{1}, \psi_{1}\right)-V\left(t, \varphi_{2}, \psi_{2}\right)\right| \leq k\left(\left|\varphi_{1}-\varphi_{2}\right|+\mid \psi_{1}-\right. \\
& \left.\psi_{2} \mid\right) ; \\
& \left(H^{\prime} 2.3\right) V_{(7)}^{\prime}(t, \varphi, \psi) \leq-a V(t, \varphi, \psi),
\end{aligned}
$$

where $a$ is a positive constant and $u(s)$ and $v(s)$ are continuous nondecreasing functions; when $s \rightarrow 0, u(s) \rightarrow 0, k$ is a positive constant. At this time, if (7) has a bounded solution $x(t, \sigma, \varphi)$ such that $|x(t, \sigma, \varphi)| \leq H_{1}$, where $t \geq \sigma \geq 0$, $H>H_{1}>0$, then (6) in $C_{H}$ has a unique almost periodic solution which is uniformly asymptotically stable.

Throughout this paper, we make the following assumptions.

(H2.1): Functions $a_{i}(u)$ are continuous bounded and there are positive constants $a_{i}^{+}, a_{i}^{-}$such that

$0<a_{i}^{-} \leq a_{i}(u) \leq a_{i}^{+}, \quad \forall u \in R, i=1,2, \ldots, m$.

(H2.2): Functions $b_{i}(u) \in C(R, R)$ and there exist positive constants $b_{i}^{-}, b_{i}^{+}$such that

$$
\begin{array}{r}
b_{i}^{-} \leq \frac{b_{i}(u)-b_{i}(v)}{u-v} \leq b_{i}^{+}, \quad u \neq v, \\
\forall u, v \in R, b_{i}(0)=0 .
\end{array}
$$

(H2.3): $c_{i j}(t), d_{i j}(t), p_{i j}(t), I_{i}(t) \in C(R, R), \tau_{i j}(t) \in$ $C\left(R, R^{+}\right)$are pseudo almost periodic functions:

$$
\begin{array}{ll}
\sup _{t \in R} c_{i j}(t)=c_{i j}^{+}>0, & \sup _{t \in R} d_{i j}(t)=d_{i j}^{+}>0, \\
\sup _{t \in R} p_{i j}(t)=p_{i j}^{+}>0, & \sup _{t \in R} I_{i}(t)=I_{i}^{+}>0,
\end{array}
$$

where $R^{+}=[0, \infty), i, j=1,2, \ldots, m$. 
$(H 2.4)$ : Delay kernel functions $G_{i j}:[0,+\infty) \rightarrow$ $[0,+\infty)$ are piecewise continuous and integrable

$$
\begin{gathered}
\int_{0}^{+\infty} G_{i j}(u) d u=1, \\
\int_{0}^{\infty} u G_{i j}(u) d u<+\infty, \\
\quad i, j=1,2, \ldots, m .
\end{gathered}
$$

(H2.5): Functions $f_{j}(u), g_{j}(u), h_{j}(u) \in C(R, R)$ satisfy the Lipschitz condition; namely, there exist nonnegative constants $L_{j}^{f}, L_{j}^{g}$, and $L_{j}^{h}$ such that

$\left|f_{j}(u)-f_{j}(v)\right| \leq L_{j}^{f}|u-v|, \quad \forall u, v \in R, j=1,2, \ldots, m$,

$\left|g_{j}(u)-g_{j}(v)\right| \leq L_{j}^{g}|u-v|, \quad \forall u, v \in R, j=1,2, \ldots, m$,

$\left|h_{j}(u)-h_{j}(v)\right| \leq L_{j}^{h}|u-v|, \quad \forall u, v \in R, j=1,2, \ldots, m$.

\section{The Existence of Pseudo Almost Periodic Solution}

In this section, we study the existence of pseudo almost periodic solution to system (1).

It follows from $(H 2.1)$ that the antiderivative of $1 / a_{i}\left(x_{i}\right)$ exists. Then we choose an antiderivative $F_{i}\left(x_{i}\right)$ of $1 / a_{i}\left(x_{i}\right)$ that satisfies $F_{i}(0)=0$. Clearly, $F_{i}^{\prime}\left(x_{i}\right)=1 / a_{i}\left(x_{i}\right)$. Because $a_{i}\left(x_{i}\right)>0, F_{i}\left(x_{i}\right)$ is increasing about $x_{i}$ and the inverse function $F_{i}^{-1}\left(x_{i}\right)$ of $F_{i}\left(x_{i}\right)$ is existential, continuous, and differential. Then $\left(F_{i}^{-1}\left(x_{i}\right)\right)^{\prime}=a_{i}\left(x_{i}\right)$. Denote $F_{i}^{\prime}\left(x_{i}\right) x_{i}^{\prime}(t)=$ $x_{i}^{\prime}(t) / a_{i}\left(x_{i}(t)\right) \doteq u_{i}^{\prime}(t)$; we get $x_{i}(t)=F_{i}^{-1}\left(u_{i}(t)\right)$. Substituting these equations into system (1), we get the following equivalent equation:

$$
\begin{aligned}
u_{i}^{\prime}(t)= & -b_{i}\left(F_{i}^{-1}\left(u_{i}(t)\right)\right) \\
& +\sum_{j=1}^{m} c_{i j}(t) f_{j}\left(F_{j}^{-1}\left(u_{j}(t)\right)\right) \\
& +\sum_{j=1}^{m} d_{i j}(t) g_{j}\left(F_{j}^{-1}\left(u_{j}\left(t-\tau_{i j}(t)\right)\right)\right) \\
& +\sum_{j=1}^{m} p_{i j}(t) \int_{-\infty}^{t} G_{i j}(t-s) h_{j}\left(F_{j}^{-1}\left(u_{j}(s)\right)\right) d s \\
& +I_{i}(t), \quad t \geq 0, \\
& u_{i}(t)=F_{i}^{-1}\left(\Phi_{i}(t)\right)=\varphi_{i}(t), \quad t<0 .
\end{aligned}
$$

From (14), we get $b_{i}\left(F_{i}^{-1}\left(u_{i}(t)\right)\right)=\left[b_{i}\left(F_{i}^{-1}\left(\theta_{i} u_{i}(t)\right)\right)\right]^{\prime} u_{i}(t) \doteq$ $b_{i}^{\sim}\left(u_{i}(t)\right) u_{i}(t)$, where $0 \leq \theta_{i} \leq 1$. Putting it into (14), we obtain

$$
\begin{aligned}
u_{i}^{\prime}(t)= & -b_{i}^{\sim}\left(u_{i}(t)\right) u_{i}(t) \\
& +\sum_{j=1}^{m} c_{i j}(t) f_{j}\left(F_{j}^{-1}\left(u_{j}(t)\right)\right) \\
& +\sum_{j=1}^{m} d_{i j}(t) g_{j}\left(F_{j}^{-1}\left(u_{j}\left(t-\tau_{i j}(t)\right)\right)\right) \\
& +\sum_{j=1}^{m} p_{i j}(t) \int_{-\infty}^{t} G_{i j}(t-s) h_{j}\left(F_{j}^{-1}\left(u_{j}(s)\right)\right) d s \\
& +I_{i}(t), \quad t \geq 0, \\
& u_{i}(t)=F_{i}^{-1}\left(\Phi_{i}(t)\right)=\varphi_{i}(t), \quad t<0 .
\end{aligned}
$$

Thus, system (1) has at least one pseudo almost periodic solution if and only if the system (15) has at least one pseudo almost periodic solution. So we only consider the pseudo almost periodic solution of system (15). By Lagrange theorem, we have

$$
\begin{aligned}
\left|F_{i}^{-1}(u)-F_{i}^{-1}(v)\right| & =\left|\left[F_{i}^{-1}\left(v+\theta_{i}(u-v)\right)\right]^{\prime}(u-v)\right| \\
& =\left|a_{i}\left(v+\theta_{i}(u-v)\right)\right||u-v| .
\end{aligned}
$$

Again by (H2.1), we get

$$
a_{i}^{-}|u-v| \leq\left|F_{i}^{-1}(u)-F_{i}^{-1}(v)\right| \leq a_{i}^{+}|u-v| .
$$

Combined with (H2.2), we have

$$
\text { (H3.6): } b_{i}^{-} a_{i}^{-} \leq b_{i}^{\prime}\left(F_{i}^{-1}(\cdot)\right) \leq b_{i}^{+} a_{i}^{+} \text {. }
$$
lemma.

In order to prove the main results, we give the following

Lemma 9. Suppose that assumptions (H2.2)-(H2.5) hold and if $\phi(t) \in P A P\left(R, R^{m}\right)$, then

$$
\begin{array}{r}
C_{i j}=\int_{-\infty}^{t} G_{i j}(t-s) \phi_{j}(s) d s \in P A P(R, R), \\
i=1,2, \ldots, m .
\end{array}
$$

Proof. From Definition 2, we have $\phi_{j}=\phi_{j 1}+\phi_{j 0}$; then

$$
\begin{aligned}
C_{i j} & =\int_{-\infty}^{t} G_{i j}(t-s) \phi_{j 1}(s) d s+\int_{-\infty}^{t} G_{i j}(t-s) \phi_{j 0}(s) d s \\
& =C_{i j 1}+C_{i j 0} .
\end{aligned}
$$

Firstly, we prove $C_{i j 1} \in A P(R, R)$. For any $\epsilon>0$, there exists a number $l=l(\epsilon)>0$ such that every interval $[a, a+l]$ 
contains at least one point of $\tau=\tau(\epsilon)$ such that $\mid \phi_{j 1}(t+\tau)-$ $\phi_{j 1}(t) \mid \leq \epsilon$ for every $t \in R$. Therefore, from $(H 2.2)-(H 2.4)$, we obtain

$$
\begin{aligned}
\mid C_{i j 1}(t & +\tau)-C_{i j 1}(t) \mid \\
= & \mid \int_{-\phi}^{t+\tau} G_{i j}(t+\tau-s) \phi_{j 1}(s) d s \\
& \quad-\int_{-\infty}^{t} G_{i j}(t-s) \phi_{j 1}(s) d s \mid \\
\leq & \int_{-\infty}^{t}\left|G_{i j}(t-s)\right|\left|\varphi_{j 1}(s+\tau)-\varphi_{j 1}(s)\right| d s \\
\leq & \epsilon \int_{-\infty}^{t}\left|G_{i j}(t-s)\right| d s \\
\leq & \epsilon
\end{aligned}
$$

so that $C_{i j 1} \in A P(R, R)$.

And then we show that $C_{i j 0} \in P A P_{0}(R, R)$ because

$$
\begin{aligned}
\lim _{T \rightarrow+\infty} & \frac{1}{2 T} \int_{-T}^{T}\left|C_{i j 0}\right| d t \\
\quad & \sup _{t \in R} \lim _{T \rightarrow+\infty} \frac{1}{2 T} \int_{-T}^{T}\left|\int_{-\infty}^{t} G_{i j}(t-s) \phi_{j 0}(s) d s\right| d t \\
& \leq \sup _{t \in R} \lim _{T \rightarrow+\infty} \frac{1}{2 T} \int_{0}^{+\infty}\left|G_{i j}(u)\right| \int_{-(T+u)}^{T+u}\left|\phi_{j 0}(v) d v\right| d u \\
& =0
\end{aligned}
$$

Thus $C_{i j 0} \in P A P_{0}(R, R)$. So $C_{i j} \in P A P(R, R)$.

Theorem 10. Suppose that (H2.1)-(H2.5) and (H3.6) hold; if

$$
\delta=\max _{1 \leq i \leq m}\left\{\frac{a_{i}^{+}}{b_{i}^{-} a_{i}^{-}} \sum_{j=1}^{m}\left(L_{j}^{f} c_{i j}^{+}+L_{j}^{g} d_{i j}^{+}+L_{j}^{h} p_{i j}^{+}\right)\right\}<1,
$$

then the system (1) has at least one pseudo almost periodic solution.

Proof. For all $z(t)=\phi(t)^{T}=\left(\phi_{1}(t), \ldots, \phi_{m}(t)\right)^{T} \in$ $\operatorname{PAP}\left(R, R^{m}\right)$, we define the nonlinear operator $T: z(t) \rightarrow$ $T(z)(t)=z_{(\phi)^{T}}(t)=\left(x_{\phi}(t)\right)^{T}$, where

$$
\begin{aligned}
x_{\phi_{i}}(t)= & \int_{-\infty}^{t} e^{-\int_{s}^{t} b_{i}^{\sim}\left(\phi_{i}(\tau)\right) d \tau} \\
& \times\left[\sum_{j=1}^{m} c_{i j}(s) f_{j}\left(F_{j}^{-1}\left(\phi_{j}(s)\right)\right)\right.
\end{aligned}
$$

$$
\begin{aligned}
& +\sum_{j=1}^{m} d_{i j}(s) g_{j}\left(F_{j}^{-1}\left(\phi_{j}\left(s-\tau_{i j}(s)\right)\right)\right) \\
& +\sum_{j=1}^{m} p_{i j}(s) \int_{-\infty}^{t} G_{i j}(s-v) h_{j}\left(F_{j}^{-1}\left(\phi_{j}(v)\right)\right) d v \\
& \left.+I_{i}(s)\right] d s .
\end{aligned}
$$

Now, we prove that

$$
T: P A P\left(R, R^{m}\right) \longrightarrow P A P\left(R, R^{m}\right)
$$

Let

$$
\begin{aligned}
E_{i j}= & \sum_{j=1}^{m} c_{i j}(s) f_{j}\left(F_{j}^{-1}\left(\phi_{j}(s)\right)\right) \\
& +\sum_{j=1}^{m} d_{i j}(s) g_{j}\left(F_{j}^{-1}\left(\phi_{j}\left(s-\tau_{i j}(s)\right)\right)\right) \\
& +\sum_{j=1}^{m} p_{i j}(s) \int_{-\infty}^{t} G_{i j}(s-v) h_{j}\left(F_{j}^{-1}\left(\phi_{j}(v)\right)\right) d v+I_{i}(s) .
\end{aligned}
$$

For $z(t) \in \operatorname{PAP}\left(R, R^{m}\right)$, conditions (H2.2)-(H2.4), Lemmas 5, 6, and 9, and the composition theorem in [16], we will get $E_{i j} \in P A P(R, R), \forall i, j=1,2, \ldots, m$.

From Definition 2, we have $E_{i j}=E_{i j 1}+E_{i j 0}, \forall i, j=$ $1,2, \ldots, m$. Where $E_{i j 1} \in A P(R, R)$ and $E_{i j 0} \in P A P_{0}(R, R)$. Then

$$
\begin{aligned}
x_{\phi_{i}}(t)= & \int_{-\infty}^{t} e^{-\int_{s}^{t} b_{i}^{\sim}\left(\phi_{i}(\tau)\right) d \tau} E_{i j 1}(s) d s \\
& +\int_{-\infty}^{t} e^{-\int_{s}^{t} b_{i}^{\sim}\left(\phi_{i}(\tau)\right) d \tau} E_{i j 0}(s) d s \\
= & T_{i j 1}+T_{i j 0},
\end{aligned}
$$

where $T_{i j 1}=\int_{-\infty}^{t} e^{-\int_{s}^{t} b_{i}^{\sim}\left(\phi_{i}(\tau)\right) d \tau} E_{i j 1}(s) d s$ and $T_{i j 0}=$ $\int_{-\infty}^{t} e^{-\int_{s}^{t} b_{i}^{\sim}\left(\phi_{i}(\tau)\right) d \tau} E_{i j 0}(s) d s$.

Because $E_{i j 1} \in A P(R, R)$, for any $\epsilon>0$, there exists a number $l=l(\epsilon)>0$ such that every interval $[a, a+l]$ contains at least one point of $\delta=\delta(\epsilon)$ such that $\sup _{t \in R} \mid E_{i j 1}(t+\delta)-$ $E_{i j 1}(t) \mid \leq \epsilon$ for every $t \in R$ and $\forall i, j=1,2, \ldots, m$. Hence, we obtain

$$
\begin{aligned}
& \left|T_{i j 1}(t+\delta)-T_{i j 1}(t)\right| \\
& \quad=\mid \int_{-\infty}^{t+\delta} e^{-\int_{s}^{t+\delta} b_{i}^{\sim}\left(\phi_{i}(\tau)\right) d \tau} E_{i j 1}(s) d s
\end{aligned}
$$




$$
\begin{aligned}
& -\int_{-\infty}^{t} e^{-\int_{s}^{t} b_{i}^{\sim}\left(\phi_{i}(\tau)\right) d \tau} E_{i j 1}(s) d s \mid \\
\leq & \int_{-\infty}^{t+\tau}\left|e^{-b_{i}^{-} a_{i}^{-}(t-s)}\right| E_{i j 1}(s+\delta)-E_{i j 1}(s) \mid d s \\
\leq & \frac{\epsilon}{b_{i}^{-} a_{i}^{-}}
\end{aligned}
$$

so that $T_{i j 1} \in A P(R, R)$.

And because

$$
\begin{aligned}
& \lim _{T \rightarrow+\infty} \frac{1}{2 T} \int_{-T}^{T}\left|\int_{-\infty}^{t} e^{-\int_{s}^{t} b_{i}^{\sim}\left(\phi_{i}(\tau)\right) d \tau} E_{i j 0}(s) d s\right| d t \\
& \leq \lim _{T \rightarrow+\infty} \frac{1}{2 T} \int_{-T}^{T}\left|\int_{-T}^{t} e^{-\int_{s}^{t} b_{i}^{\sim}\left(\phi_{i}(\tau)\right) d \tau} E_{i j 0}(s) d s\right| d t \\
& +\lim _{T \rightarrow+\infty} \frac{1}{2 T} \int_{-T}^{T}\left|\int_{-\infty}^{-T} e^{-\int_{s}^{t} b_{i}^{\sim}\left(\phi_{i}(\tau)\right) d \tau} E_{i j 0}(s) d s\right| d t \\
& \leq \lim _{T \rightarrow+\infty} \frac{1}{2 T} \int_{-T}^{T}\left\|E_{i j 0}(t)\right\| d t \int_{-T}^{t} e^{-b_{i}^{-} a_{i}^{-}(t-s)} d s \\
& +\lim _{T \rightarrow+\infty} \frac{\sup _{t \in R}\left|E_{i j 0}(t)\right|}{2 T} \int_{-T}^{T} d t\left(\int_{-\infty}^{-T}\left|e^{-b_{i}^{-} a_{i}^{-}(t-s)}\right| d s\right) \\
& \leq \lim _{T \rightarrow+\infty} \frac{1}{2 T b_{i}^{-} a_{i}^{-}} \int_{-T}^{T}\left\|E_{i j 0}(t)\right\| d t \\
& +\lim _{T \rightarrow+\infty} \frac{\sup _{t \in R}\left|E_{i j 0}(t)\right|}{2 T\left(b_{i}^{-} a_{i}^{-}\right)^{2}}\left(1-e^{-b_{i}^{-} a_{i}^{-}(2 T)}\right) \\
& =0+\lim _{T \rightarrow+\infty} \frac{\sup _{t \in R}\left|E_{i j 0}(t)\right|}{2 T\left(b_{i}^{-} a_{i}^{-}\right)^{2}}\left(1-e^{-b_{i}^{-} a_{i}^{-}(2 T)}\right) \\
& =0 \text {, }
\end{aligned}
$$

thus $T_{i j 0} \in P A P_{0}(R, R)$. So $\forall i, j=1,2, \ldots, m, x_{\phi_{i}}(t) \in$ $\operatorname{PAP}(R, R)$. Therefore $z_{(\phi)^{T}}(t) \in \operatorname{PAP}\left(R, R^{m}\right)$.

From Lemma 9, $X=P A P\left(R, R^{m}\right)$ is a Banach space. If

$$
\delta<1
$$

then there exists a sufficiently large $\beta \geq 1$ such that

$$
\delta \leq 1-\beta^{-1} I,
$$

where

$$
I=\max _{1 \leq i \leq m}\left\{\frac{I_{i}^{+}}{b_{i}^{-} a_{i}^{-}}\right\} .
$$

We choose a closed subset

$$
B=\left\{z(t)=\phi(t)^{T}=\left(\phi_{1}(t), \ldots, \phi_{m}(t)\right)^{T} \in X:\|z\| \leq \beta\right\} .
$$

Firstly, we prove that $T: B \rightarrow B$; that is, $T B \subset B$.
From (29)-(32) and for $\forall z \in B$, we get

$$
\begin{aligned}
\left|x_{\phi_{i}}(t)\right| \leq & \max _{1 \leq i \leq m}\left\{\frac{a_{i}^{+}}{b_{i}^{-} a_{i}^{-}} \sum_{j=1}^{n}\left(L_{j}^{f} c_{i j}^{+}+L_{j}^{g} d_{i j}^{+}+L_{j}^{h} p_{i j}^{+}\right)\right\} \\
& \times\|z\|+\max _{1 \leq i \leq m}\left\{\frac{I_{i}^{+}}{b_{i}^{-} a_{i}^{-}}\right\} \leq \beta \delta+I \leq \beta .
\end{aligned}
$$

Secondly, we prove that the mapping $T$ is completely continuous.

By the continuity of the function $f_{j}, g_{j}, h_{j}$, for any $\varepsilon>0$, there is $\gamma=\gamma(\varepsilon, \beta)$ such that

$$
\begin{array}{r}
\left|f_{j}(u)-f_{j}(v)\right| \leq \frac{L_{j}^{f} \varepsilon}{\delta}, \quad|u-v| \leq \gamma, \\
\forall u, v \in B, \quad j=1,2, \ldots, m, \\
\left|g_{j}(u)-g_{j}(v)\right| \leq \frac{L_{j}^{g} \varepsilon}{\delta}, \quad|u-v| \leq \gamma, \\
\forall u, v \in B, \quad j=1,2, \ldots, m, \\
\left|h_{j}(u)-h_{j}(v)\right| \leq \frac{L_{j}^{h} \varepsilon}{\delta}, \quad|u-v| \leq \gamma, \\
\forall u, v \in B, \quad j=1,2, \ldots, m .
\end{array}
$$

Let $w(t)=\left(\psi_{1}(t), \ldots, \psi_{m}(t)\right), z, w \in B$, and $\|z-w\| \leq \gamma$; then $\|z\| \leq \gamma,\|w\| \leq \gamma$ and $\phi_{j}(t), \psi_{j}(t) \in C(R, B)$; then, for any $s \in R$, we get $\left|\phi_{j}(s)-\psi_{1}(s)\right| \leq \gamma$. So, we have

$$
\begin{aligned}
& \left|f_{j}\left(F_{j}^{-1}\left(\phi_{j}(s)\right)\right)-f_{j}\left(F_{j}^{-1}\left(\psi_{j}(s)\right)\right)\right| \leq \frac{L_{j}^{f} \varepsilon}{\delta}, \quad|u-v| \leq \gamma, \\
& \left|g_{j}\left(F_{j}^{-1}\left(\phi_{j}(s)\right)\right)-f_{j}\left(F_{j}^{-1}\left(\psi_{j}(s)\right)\right)\right| \leq \frac{L_{j}^{g} \varepsilon}{\delta}, \quad|u-v| \leq \gamma, \\
& \left|h_{j}\left(F_{j}^{-1}\left(\phi_{j}(s)\right)\right)-f_{j}\left(F_{j}^{-1}\left(\psi_{j}(s)\right)\right)\right| \leq \frac{L_{j}^{h} \varepsilon}{\delta} . \quad|u-v| \leq \gamma .
\end{aligned}
$$

Thus

$\|T(z)(t)-T(w)(t)\|$

$$
\begin{aligned}
& \leq \max _{1 \leq i \leq m}\left\{\frac{1}{b_{i}^{-} a_{i}^{-}} \sum_{j=1}^{m} a_{i}^{+}\left(L_{j}^{f} c_{i j}^{+}+L_{j}^{g} d_{i j}^{+}+L_{j}^{h} p_{i j}^{+}\right)\right\} \frac{\varepsilon}{\delta} \\
& \leq \delta \frac{\varepsilon}{\delta} \leq \varepsilon .
\end{aligned}
$$

Therefore, $T$ is continuous.

Thirdly, we show that $T$ is compact.

Let $S=\{z(t) \in X:\|z\| \leq K\}$, where $K>0$ to be any constant. We denote $\rho=\max _{1 \leq i \leq m}\left\{\left(a_{i}^{+} / b_{i}^{-} a_{i}^{-}\right) \sum_{j=1}^{m} K\left(L_{j}^{f} c_{i j}^{+}+\right.\right.$ $\left.\left.L_{j}^{g} d_{i j}^{+}+L_{j}^{h} p_{i j}^{+}\right)+I_{i}^{+}\right\}$. Then we have

$$
\|T(z)(t)\|=\sup _{t \in R} \max _{1 \leq i \leq m}\left|x_{\phi_{i}}(t)\right| \leq \rho, \quad \forall z \in S .
$$


Hence, $T$ is uniformly bounded. Then, from (23), we get

$$
\begin{aligned}
{\left[\left|x_{\phi_{i}}(t)\right|\right]^{\prime}=} & -b_{i}^{\sim}\left(x_{\phi_{i}}(t)\right) x_{\phi_{i}}(t) \\
& +\sum_{j=1}^{m} c_{i j}(t) f_{j}\left(F_{j}^{-1}\left(\phi_{j}(t)\right)\right) \\
& +\sum_{j=1}^{m} d_{i j}(t) g_{j}\left(F_{j}^{-1}\left(\phi_{j}\left(t-\tau_{i j}(t)\right)\right)\right) \\
& +\sum_{j=1}^{m} p_{i j}(t) \int_{-\infty}^{t} G_{i j}(t-s) h_{j}\left(F_{j}^{-1}\left(\phi_{j}(s)\right)\right) d s \\
& +I_{i}(t) \\
\leq & b_{i}^{+} \rho+\sum_{j=1}^{m} a_{j}^{+} K\left(L_{j}^{f} c_{i j}^{+}+L_{j}^{g} d_{i j}^{+}+L_{j}^{h} p_{i j}^{+}\right)+I_{i}^{+} \leq L,
\end{aligned}
$$

where

$$
L=\max _{1 \leq i \leq m}\left\{b_{i}^{+} \rho+\sum_{j=1}^{m} a_{j}^{+} K\left(L_{j}^{f} c_{i j}^{+}+L_{j}^{g} d_{i j}^{+}+L_{j}^{h} p_{i j}^{+}\right)+I_{i}^{+}\right\} .
$$

Therefore, $T$ is equicontinuous. By the Ascoli-Arzela theorem, the operator $T$ is compact; then it is completely continuous. By the Schauder fixed point theorem, the system (1) has at least one pseudo almost periodic solution.

\section{The Global Uniform Asymptotic Stability of Pseudo Almost Periodic Solution}

In order to discuss the global uniform asymptotic stability of pseudo almost periodic solution to system (1), we give the following assumptions:

(H4.1): delay functions $\tau_{i j}(t) \in C^{1}\left(R, R^{+}\right)$satisfy that $\dot{\tau}_{i j}(t) \leq \tau_{i j}^{*}<1, i, j=1,2, \ldots, m$;

(H4.2): $N=\min _{1 \leq i \leq m}\left\{N_{i}\right\}>0$, where $N_{i}=b_{i}^{-}-$ $\sum_{j=1}^{m} c_{i j}^{+} L_{j}^{f}-\sum_{j=1}^{m}\left(d_{i j}^{+} L_{j}^{g} /\left(1-\tau_{i j}^{*}\right)\right)-\sum_{j=1}^{m} p_{i j}^{+} L_{j}^{h}$.

Theorem 11. Assume that (H2.1)-(H2.5) and (H4.1)-(H4.2) hold; then the pseudo almost periodic solution of system (1) is globally uniformly asymptotically stable.

Proof. The product system of the system (1) is

$$
\begin{aligned}
x_{i}^{\prime}(t)= & -a_{i}\left(x_{i}(t)\right) \\
& \times\left[b_{i}\left(x_{i}(t)\right)-\sum_{j=1}^{m} c_{i j}(t) f_{j}\left(x_{j}(t)\right)\right.
\end{aligned}
$$

$$
\begin{aligned}
& -\sum_{j=1}^{m} d_{i j}(t) g_{j}\left(x_{j}\left(t-\tau_{i j}(t)\right)\right) \\
& \left.-\sum_{j=1}^{m} p_{i j}(t) \int_{-\infty}^{t} G_{i j}(t-s) h_{j}\left(x_{j}(s)\right) d s-I_{i}(t)\right], \\
& y_{i}^{\prime}(t)=-a_{i}\left(y_{i}(t)\right) \\
& \times\left[b_{i}\left(y_{i}(t)\right)-\sum_{j=1}^{m} c_{i j}(t) f_{j}\left(y_{j}(t)\right)\right. \\
& -\sum_{j=1}^{m} d_{i j}(t) g_{j}\left(y_{j}\left(t-\tau_{i j}(t)\right)\right) \\
& \left.-\sum_{j=1}^{m} p_{i j}(t) \int_{-\infty}^{t} G_{i j}(t-s) h_{j}\left(y_{j}(s)\right) d s-I_{i}(t)\right] .
\end{aligned}
$$

In order to apply the conclusion of Lemma 8, we construct a Lyapunov functional about product system (40)

$$
V(t)=V_{1}(t)+V_{2}(t)+V_{3}(t)
$$

where

$$
\begin{gathered}
V_{1}(t)=\sum_{i=1}^{m}\left|\int_{y_{i}(t)}^{x_{i}(t)} \frac{1}{a_{i}(s)} d s\right| ; \\
V_{2}(t)=\sum_{i=1}^{m} \sum_{j=1}^{m} \frac{d_{i j}^{+} L_{j}^{g}}{1-\tau_{i j}^{*}} \int_{t-\tau_{i j}(t)}^{t}\left|x_{i}(s)-y_{i}(s)\right| d s ; \\
V_{3}(t)=\sum_{i=1}^{m} \sum_{j=1}^{m} p_{i j}^{+} L_{j}^{h} \int_{0}^{+\infty} G_{i j}(s) \int_{t-s}^{t}\left|x_{i}(u)-y_{i}(u)\right| d u d s .
\end{gathered}
$$

Let $X(t)=x(t)^{T}=\left(x_{1}(t), \ldots, x_{m}(t)\right)^{T}$ and $Y(t)=y(t)^{T}=$ $\left(y_{1}(t), \ldots, y_{m}(t)\right)^{T}$. For product system $(X, Y)$, we receive

$|X-Y| \leq V(t, X(t), Y(t))$

$$
\begin{aligned}
& \leq \sum_{i=1}^{m}\left\{\frac{1}{a_{i}^{-}}+\sum_{j=1}^{m} \frac{d_{i j}^{+} L_{j}^{g} \tau_{i j}^{+}}{1-\tau_{i j}^{*}}+\sum_{j=1}^{n} p_{i j}^{+} L_{i}^{h} \int_{0}^{+\infty} G_{i j}(s) s d s\right\} \\
& \times\left|x_{i}(t)-y_{i}(t)\right| \\
& \leq M_{i} \sum_{i=1}^{m}\left|x_{i}(t)-y_{i}(t)\right| \\
& \leq M|X-Y|,
\end{aligned}
$$

where $M_{i}=\left(1 / a_{i}^{-}\right)+\sum_{j=1}^{m}\left(d_{i j}^{+} L_{j}^{g} \tau_{i j}^{+} /\left(1-\tau_{i j}^{*}\right)\right)+$ $\sum_{j=1}^{n} p_{i j}^{+} L_{i}^{h} \int_{0}^{+\infty} G_{i j}(s) s d s$ and $M=\max _{1 \leq i \leq m}\left\{M_{i}\right\}$. Let 
$u(s)=s$ and $v(s)=M s$; we easily know it satisfies condition $\left(H^{\prime} 2.1\right)$ of Lemma 8. Then we obtain

$$
\begin{aligned}
\mid V( & t, X, Y)-V\left(t, X^{*}, Y^{*}\right) \mid \\
= & \left|\sum_{i=1}^{m}\right| \int_{y_{i}(t)}^{x_{i}(t)} \frac{1}{a_{i}(s)} d s\left|-\sum_{i=1}^{m}\right| \int_{y_{i}^{*}(t)}^{x_{i}^{*}(t)} \frac{1}{a_{i}(s)} d s \mid \\
& +\sum_{i=1}^{m} \sum_{j=1}^{m} \frac{d_{i j}^{+} L_{j}^{g}}{1-\tau_{i j}^{*}} \int_{t-\tau_{i j}(t)}^{t}\left|x_{i}(s)-y_{i}(s)\right| d s \\
& -\sum_{i=1}^{m} \sum_{j=1}^{m} \frac{d_{i j}^{+} L_{j}^{g}}{1-\tau_{i j}^{*}} \int_{t-\tau_{i j}(t)}^{t}\left|x_{i}^{*}(s)-y_{i}^{*}(s)\right| d s \\
& -\sum_{i=1}^{m} \sum_{j=1}^{m} p_{i j}^{+} L_{j}^{h} \int_{0}^{+\infty} G_{i j}(s) \int_{t-s}^{t}\left|x_{i}(u)-y_{i}(u)\right| d u d s \\
& -\sum_{i=1}^{m} \sum_{j=1}^{m} p_{i j}^{+} L_{j}^{h} \int_{0}^{+\infty} G_{i j}(s) \int_{t-s}^{t}\left|x_{i}^{*}(u)-y_{i}^{*}(u)\right| d u d s \mid \\
\leq & \sum_{i=1}^{m} \frac{1}{a_{i}^{-}}\left|x_{i}(t)-x_{i}^{*}(t)\right|+\sum_{i=1}^{m} \frac{1}{a_{i}^{-}}\left|y_{i}(t)-y_{i}^{*}(t)\right| \\
& +\sum_{i=1}^{m} \sum_{j=1}^{m} \frac{d_{i j}^{+} L_{j}^{g}}{1-\tau_{i j}^{*}} \\
& \times \int_{t-\tau_{i j}(t)}^{t}\left(\left|x_{i}(s)-x_{i}^{*}(s)\right|+\left|y_{i}(s)-y_{i}^{*}(s)\right|\right) d s \\
& +\sum_{i=1}^{m} \sum_{j=1}^{m} p_{i j}^{+} L_{j}^{h} \int_{0}^{+\infty} G_{i j}(s) \\
& M\left(\left|X-X^{*}\right|+\left|x_{i}(t)-x_{i}^{*}(t)\right|+\left|y_{i}(t)-y_{i}^{*}(t)\right|\right) \\
&
\end{aligned}
$$

We also know that it satisfies condition $\left(H^{\prime} 2.2\right)$ of Lemma 8 .

Calculating the upright derivative of $V_{1}(t), V_{2}(t)$, and $V_{3}(t)$ along the system (40), respectively, and noting that $\left(\left(1-\dot{\tau}_{i j}(t)\right) /\left(1-\tau_{i j}^{*}\right)\right)>1$, we have

$$
\begin{aligned}
& \left.D^{+} V_{1}(t)\right|_{(40)} \\
& =\sum_{i=1}^{m} \operatorname{sgn}\left(x_{i}(t)-y_{i}(t)\right)\left[\frac{x_{i}^{\prime}(t)}{a_{i}\left(x_{i}(t)\right)}-\frac{y_{i}^{\prime}(t)}{a_{i}\left(y_{i}(t)\right)}\right] \\
& \leq \sum_{i=1}^{m}\left\{-\frac{b_{i}\left(x_{i}(t)\right)-b_{i}\left(y_{i}(t)\right)}{x_{i}(t)-y_{i}(t)}\left|x_{i}(t)-y_{i}(t)\right|\right. \\
& \quad+\sum_{j=1}^{m} c_{i j}^{+} L_{j}^{f}\left|x_{j}(t)-y_{j}(t)\right|
\end{aligned}
$$

$$
\begin{aligned}
& +\sum_{j=1}^{m} d_{i j}^{+} L_{j}^{g}\left|x_{j}\left(t-\tau_{i j}(t)\right)-y_{j}\left(t-\tau_{i j}(t)\right)\right| \\
& \left.+\sum_{j=1}^{n} p_{i j}^{+} L_{j}^{h} \int_{0}^{+\infty} G_{i j}(s)\left|x_{j}(t-s)-y_{j}(t-s)\right| d s\right\} \\
& \leq \sum_{i=1}^{m}\left\{-b_{i}^{-}\left|x_{i}(t)-y_{i}(t)\right|+\sum_{j=1}^{m} c_{i j}^{+} L_{j}^{f}\left|x_{j}(t)-y_{j}(t)\right|\right. \\
& +\sum_{j=1}^{m} d_{i j}^{+} L_{j}^{g}\left|x_{j}\left(t-\tau_{i j}(t)\right)-y_{j}\left(t-\tau_{i j}(t)\right)\right| \\
& \left.+\sum_{j=1}^{n} p_{i j}^{+} L_{j}^{h} \int_{0}^{+\infty} G_{i j}(s)\left|x_{j}(t-s)-y_{j}(t-s)\right| d s\right\} ;
\end{aligned}
$$$$
\left.D^{+} V_{2}(t)\right|_{(40)}
$$$$
=\sum_{i=1}^{m} \sum_{j=1}^{m} \frac{d_{i j}^{+} L_{j}^{g}}{1-\tau_{i j}^{*}}\left|x_{i}(t)-y_{i}(t)\right|
$$$$
-\sum_{i=1}^{m} \sum_{j=1}^{m} \frac{d_{i j}^{+} L_{j}^{g}}{1-\tau_{i j}^{*}}\left|x_{i}\left(t-\tau_{i j}(t)\right)-y_{i}\left(t-\tau_{i j}(t)\right)\right| ;
$$$$
\left.D^{+} V_{3}(t)\right|_{(40)}
$$$$
=\sum_{i=1}^{m} \sum_{j=1}^{m} p_{i j}^{+} L_{j}^{h} \int_{0}^{+\infty} G_{i j}(s)\left|x_{i}(t)-y_{i}(t)\right| d s
$$$$
-\sum_{i=1}^{m} \sum_{j=1}^{m} p_{i j}^{+} L_{j}^{f} \int_{0}^{+\infty} G_{i j}(s)\left|x_{i}(t-s)-y_{i}(t-s)\right| d s .
$$

Combining (45) and assumptions (H4.1) and (H4.2), we get

$$
\begin{aligned}
\left.D^{+} V(t)\right|_{(40)} \\
\leq \sum_{i=1}^{m}\left\{-b_{i}^{-}+\sum_{j=1}^{m} c_{i j}^{+} L_{j}^{f}+\sum_{j=1}^{m} \frac{d_{i j}^{+} L_{j}^{g}}{1-\tau_{i j}^{*}}+\sum_{j=1}^{m} p_{i j}^{+} L_{j}^{h}\right\} \\
\quad \times\left|x_{i}(t)-y_{i}(t)\right| \\
\leq-N \sum_{j=1}^{m}\left|x_{i}(t)-y_{i}(t)\right| \\
\leq-a V(t) .
\end{aligned}
$$

From assumption (H4.2), we have $a=N / M>0$.

By Lemma 8, the pseudo almost periodic solutions of system (1) are globally uniformly asymptotically stable. This completes the proof. 
Corollary 12. Consider the following periodic CGNNs systems:

$$
\begin{aligned}
x_{i}^{\prime}(t)= & -a_{i}\left(x_{i}(t)\right) \\
& \times\left[b_{i}\left(x_{i}(t)\right)-\sum_{j=1}^{m} c_{i j}(t) f_{j}\left(x_{j}(t)\right)\right. \\
& -\sum_{j=1}^{m} d_{i j}(t) f_{j}\left(x_{j}\left(t-\tau_{i j}(t)\right)\right) \\
& \left.-\sum_{j=1}^{m} p_{i j}(t) \int_{-\infty}^{t} G_{i j}(t-s) f_{j}\left(x_{j}(s)\right) d s-I_{i}(t)\right], \\
& \quad x_{i}(t)=\Phi_{i}(t), \quad t<0,
\end{aligned}
$$

where $i=1,2, \ldots, m$, and the following assumptions hold.

(G4.1): Functions $a_{i}(u)$ are continuous bounded and there are positive constants $a_{i}^{+}, a_{i}^{-}$such that

$$
0<a_{i}^{-} \leq a_{i}(u) \leq a_{i}^{+}, \quad \forall u \in R, i=1,2, \ldots, m .
$$

(G4.2): Functions $b_{i}(u) \in C(R, R)$ and there exist positive constants $b_{i}^{-}, b_{i}^{+}$such that

$$
\begin{array}{r}
b_{i}^{-} \leq \frac{b_{i}(u)-b_{i}(v)}{u-v} \leq b_{i}^{+}, \quad u \neq v, \\
\forall u, v \in R, \quad b_{i}(0)=0 .
\end{array}
$$

(G4.3): $c_{i j}(t), d_{i j}(t)$ and $p_{i j}(t), I_{i}(t) \in C(R, R), \tau_{i j}(t) \in$ $C\left(R, R^{+}\right)$are all periodic functions, and

$$
\begin{array}{ll}
\sup _{t \in R} c_{i j}(t)=c_{i j}^{+}>0, & \sup _{t \in R} d_{i j}(t)=d_{i j}^{+}>0, \\
\sup _{t \in R} p_{i j}(t)=p_{i j}^{+}>0, & \sup _{t \in R} I_{i}(t)=I_{i}^{+}>0,
\end{array}
$$

where $R^{+}=[0, \infty), i, j=1,2, \ldots, m$.

(G4.4): Delay kernel functions $G_{i j}:[0,+\infty) \rightarrow$ $[0,+\infty)$ are piecewise continuous and integrable

$$
\begin{array}{r}
\int_{0}^{+\infty} G_{i j}(u) d u=1, \int_{0}^{\infty} u G_{i j}(u) d u<+\infty, \\
i, j=1,2, \ldots, m .
\end{array}
$$

(G4.5): Functions $f_{j}(u) \in C(R, R)$ satisfy the Lipschitz condition; namely, there exist nonnegative constants $L_{j}$ such that

$$
\begin{gathered}
\left|f_{j}(u)-f_{j}(v)\right| \leq L_{j}|u-v| \\
\forall u, v \in R, \quad j=1,2, \ldots, m
\end{gathered}
$$

(G4.6): If $\delta=\max _{1 \leq i \leq m}\left\{\left(a_{i}^{+} / b_{i}^{-} a_{i}^{-}\right) \sum_{j=1}^{m} L_{j}\left(c_{i j}^{+}+d_{i j}^{+}+\right.\right.$ $\left.\left.p_{i j}^{+}\right)\right\}<1$,

then the system (47) has at least one periodic solution.

Corollary 13. Assume that (G4.1)-(G4.6) hold and suppose further that

(G4.7): delay functions $\tau_{i j}(t) \in C^{1}\left(R, R^{+}\right)$satisfy that $\dot{\tau}_{i j}(t) \leq \tau_{i j}^{*}<1, i, j=1,2, \ldots, m$.

(G4.8): $N=\min _{1 \leq i \leq m}\left\{N_{i}\right\}>0$, where $N_{i}=b_{i}^{-}-$ $\sum_{j=1}^{m} c_{i j}^{+} L_{j}-\sum_{j=1}^{m}\left(d_{i j}^{+} L_{j} /\left(1-\tau_{i j}^{*}\right)\right)-\sum_{j=1}^{m} p_{i j}^{+} L_{j}$; then the periodic solution of system (47) is globally uniformly asymptotically stable.

Remark 14. Recently, the global exponential stability of periodic or almost periodic solution to CGNNs is studied by many scholars (see [15-30]). However, few authors pay attention to the global uniform asymptotic stability. Corollaries 12 and 13 provide some new results.

\section{An Example}

An example is given to illustrate the feasibility of main results in this paper. Consider the following simple neural networks:

$$
\begin{aligned}
x_{i}^{\prime}(t)=- & a_{i}\left(x_{i}(t)\right) \\
& \times\left[b_{i}\left(x_{i}(t)\right)-\sum_{j=1}^{2} c_{i j}(t) f_{j}\left(x_{j}(t)\right)\right. \\
& -\sum_{j=1}^{2} d_{i j}(t) g_{j}\left(x_{j}\left(t-\tau_{i j}(t)\right)\right) \\
& \left.-\sum_{j=1}^{2} p_{i j}(t) \int_{-\infty}^{t} G_{i j}(t-s) h_{j}\left(x_{j}(s)\right) d s-I_{i}(t)\right], \\
& \\
& t \geq 0, i=1,2,
\end{aligned}
$$

where the initial functions $x_{1}(t)=4+\cos (\pi t) 1, t<0, x_{2}(t)=$ $5+\sin (2 t), t<0 . a_{i}\left(x_{i}(t)\right)=4+\cos \pi t-e^{-\left|x_{i}(t)\right|}, b_{i}\left(x_{i}(t)\right)=$ $5+\sin 2 t-e^{-\left|x_{i}(t)\right|}$. Let

$$
\begin{aligned}
& \left(\begin{array}{lll}
c_{11}(t) & c_{12}(t) \\
c_{21} & (t) & c_{22}(t)
\end{array}\right) \\
& \quad=\frac{1}{14}\left(\begin{array}{cc}
\cos t+e^{-t^{4} \cos ^{4} t} & \cos (\sqrt{2} t)+e^{-t^{4} \sin ^{4} t} \\
\cos (\sqrt{5} t)+e^{-t^{4} \cos ^{4} t} & \sin (2 t)+e^{-t^{2} \cos ^{4} t}
\end{array}\right),
\end{aligned}
$$




$$
\begin{aligned}
& \left(\begin{array}{ll}
d_{11}(t) & d_{12}(t) \\
d_{21}(t) & d_{22}(t)
\end{array}\right) \\
& =\frac{1}{14}\left(\begin{array}{cc}
\sin t+e^{-t^{2} \cos ^{2} t} & \cos (\sqrt{4} t)+e^{-t^{2} \cos ^{4} t} \\
\sin (\sqrt{3} t)+e^{-t^{4} \cos ^{4} t} & \sin (2 t)+e^{-t^{4} \cos ^{4} t}
\end{array}\right), \\
& \left(\begin{array}{ll}
p_{11}(t) & p_{12}(t) \\
p_{21}(t) & p_{22}(t)
\end{array}\right) \\
& =\frac{1}{14}\left(\begin{array}{ll}
\cos (\sqrt{5} t)+e^{-t^{4} \cos ^{2} t} & \sin (\sqrt{3} t)+e^{-t^{4} \cos ^{4} t} \\
\cos (\sqrt{3} t)+e^{-t^{2} \cos ^{2} t} & \sin (2 t)+e^{-t^{2} \cos ^{4} t}
\end{array}\right) .
\end{aligned}
$$

$I_{1}(t)=I_{2}(t)=2\left(\sin (\sqrt{3} t)+e^{-t^{2} \cos ^{4} t}\right), f_{j}\left(x_{j}\right)=g_{j}\left(x_{j}\right)=$ $h_{j}\left(x_{j}\right)=((|x+1|-|x-1|) / 2), G_{i j}(u)=e^{-u}, \tau_{i j}^{*}=4 / 5$. Then, we have $a_{i}^{+}=5, a_{i}^{-}=2, b_{i}^{-}=3, c_{i j}^{+}=d_{i j}^{+}=p_{i j}^{+}=1 / 7$, $L_{j}^{f}=L_{j}^{g}=L_{j}^{h}=1$, where $i, j=1,2$. Moreover

$$
\begin{aligned}
& \delta=\max _{1 \leq i \leq 2}\left\{\frac{a_{i}^{+}}{b_{i}^{-} a_{i}^{-}} \sum_{j=1}^{2}\left(L_{j}^{f} c_{i j}^{+}+L_{j}^{g} d_{i j}^{+}+L_{j}^{h} p_{i j}^{+}\right)\right\}=\frac{5}{7}<1, \\
& N_{i}=b_{i}^{-}-\sum_{j=1}^{2} c_{i j}^{+} L_{j}^{f}-\sum_{j=1}^{2} \frac{d_{i j}^{+} L_{j}^{g}}{1-\tau_{i j}^{*}}-\sum_{j=1}^{2} p_{i j}^{+} L_{j}^{h}=1>0, \\
& i=1,2 .
\end{aligned}
$$

Thus, by Theorem 10, we know that system (53) has at least one pseudo almost periodic solution. It follows from Theorem 11 that the unique pseudo almost periodic solution of system (53) is globally uniformly asymptotically stable (Figure 1).

\section{Conclusions}

In this paper, the existence and uniform asymptotic stability of pseudo almost periodic solutions of system (1) is discussed. By applying Schauder fixed point theorem and constructing a suitable Lyapunov functional, some sufficient conditions are obtained to ensure the existence and uniform asymptotic stability of pseudo almost periodic solutions of system (1). The results have important leading significance in the design and applications of CGNNs. In addition, an example is given to demonstrate the effectiveness of main results.

\section{Conflict of Interests}

The authors declare that there is no conflict of interests regarding the publication of this paper.

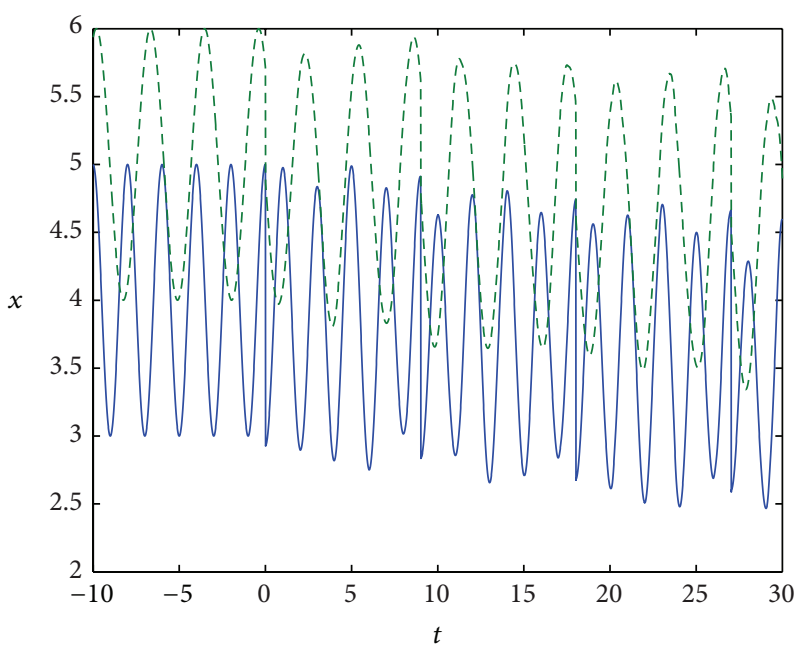

FIGURE 1: Simulated results of the solutions.

\section{Acknowledgments}

This work was supported by the National Natural Science Foundation of China (11361010) and Scientific Research Project in Guangxi of China Department of Education (201106LX613).

\section{References}

[1] M. A. Cohen and S. Grossberg, "Absolute stability of global pattern formation and parallel memory storage by competitive neural networks," IEEE Transactions on Systems, Man, and Cybernetics, vol. 13, no. 5, pp. 815-826, 1983.

[2] X. Fu and X. Li, "LMI conditions for stability of impulsive stochastic Cohen-Grossberg neural networks with mixed delays," Communications in Nonlinear Science and Numerical Simulation, vol. 16, no. 1, pp. 435-454, 2011.

[3] X. Huang, J. Cao, and D. W. C. Ho, "Existence and attractivity of almost periodic solution for recurrent neural networks with unbounded delays and variable coefficients," Nonlinear Dynamics, vol. 45, no. 3-4, pp. 337-351, 2006.

[4] K. Li, "Stability analysis for impulsive Cohen-Grossberg neural networks with time-varying delays and distributed delays," Nonlinear Analysis: Real World Applications, vol. 10, no. 5, pp. 2784-2798, 2009.

[5] J. J. Oliveira, "Global stability of a Cohen-Grossberg neural network with both time-varying and continuous distributed delays," Nonlinear Analysis: Real World Applications, vol. 12, no. 5, pp. 2861-2870, 2011.

[6] J.-L. Wang, H.-N. Wu, and L. Guo, "Stability analysis of reaction-diffusion Cohen-Grossberg neural networks under impulsive control," Neurocomputing, vol. 106, no. 15, pp. 21-30, 2013.

[7] J.-L. Wang, H.-N. Wu, and L. Guo, "Passivity and stability analysis of reaction-diffusion neural networks with dirichlet boundary conditions," IEEE Transactions on Neural Networks, vol. 22, no. 12, pp. 2105-2116, 2011.

[8] L. Wang, "Stability of Cohen-Grossberg neural networks with distributed delays," Applied Mathematics and Computation, vol. 160, no. 1, pp. 93-110, 2005. 
[9] H. Zhao, L. Chen, and Z. Mao, "Existence and stability of almost periodic solution for Cohen-Grossberg neural networks with variable coefficients," Nonlinear Analysis: Real World Applications, vol. 9, no. 2, pp. 663-673, 2008.

[10] Q. Zhu and J. Cao, "Exponential stability analysis of stochastic reaction-diffusion Cohen-Grossberg neural networks with mixed delays," Neurocomputing, vol. 74, no. 17, pp. 3084-3091, 2011.

[11] L. V. Hien, T. T. Loan, B. T. H. Trang, and H. Trinh, "Existence and global asymptotic stability of positive periodic solution of delayed Cohen-Grossberg neural networks," Applied Mathematics and Computation, vol. 240, pp. 200-212, 2014.

[12] C.-H. Li and S.-Y. Yang, "Existence and attractivity of periodic solutions to non-autonomous Cohen-Grossberg neural networks with time delays," Chaos, Solitons \& Fractals, vol. 41, no. 3, pp. 1235-1244, 2009.

[13] X. Liao and C. Li, "Global attractivity of Cohen-Grossberg model with finite and infinite delays," Journal of Mathematical Analysis and Applications, vol. 315, no. 1, pp. 244-262, 2006.

[14] L. Wan and Q. Zhou, "Asymptotic behaviors of stochastic Cohen-Grossberg neural networks with mixed time-delays," Applied Mathematics and Computation, vol. 225, pp. 541-549, 2013.

[15] C. Bai, "Global exponential stability and existence of periodic solution of Cohen-Grossberg type neural networks with delays and impulses," Nonlinear Analysis: Real World Applications, vol. 9, no. 3, pp. 747-761, 2008.

[16] F. Chérif, "Existence and global exponential stability of pseudo almost periodic solution for SICNNs with mixed delays," Journal of Applied Mathematics and Computing, vol. 39, no. 12, pp. 235-251, 2012.

[17] X. Chen and Q. Song, "Global exponential stability of the periodic solution of delayed Cohen-Grossberg neural networks with discontinuous activations," Neurocomputing, vol. 73, no. 16-18, pp. 3097-3104, 2010.

[18] T. Liang, Y. Yang, Y. Liu, and L. Li, "Existence and global exponential stability of almost periodic solutions to CohenGrossberg neural networks with distributed delays on time scales," Neurocomputing, vol. 123, no. 10, pp. 207-215, 2014.

[19] Y. Li and X. Fan, "Existence and global exponential stability of almost periodic solution for Cohen-Grossberg BAM neural networks with variable coefficients," Applied Mathematical Modelling, vol. 33, no. 4, pp. 2114-2120, 2009.

[20] B. Li and D. Xu, "Existence and exponential stability of periodic solution for impulsive Cohen-Grossberg neural networks with time-varying delays," Applied Mathematics and Computation, vol. 219, no. 5, pp. 2506-2520, 2012.

[21] F. Long, Y. Wang, and S. Zhou, "Existence and exponential stability of periodic solutions for a class of Cohen-Grossberg neural networks with bounded and unbounded delays," Nonlinear Analysis: Real World Applications, vol. 8, no. 3, pp. 797-810, 2007.

[22] S. Mandal and N. C. Majee, "Existence of periodic solutions for a class of Cohen-Grossberg type neural networks with neutral delays," Neurocomputing, vol. 74, no. 6, pp. 1000-1007, 2011.

[23] F. Ren and J. Cao, "Periodic solutions for a class of higher-order Cohen-Grossberg type neural networks with delays," Computers and Mathematics with Applications, vol. 54, no. 6, pp. 826-839, 2007.

[24] D. Wang and L. Huang, "Almost periodic dynamical behaviors for generalized Cohen-Grossberg neural networks with discontinuous activations via differential inclusions," Communications in Nonlinear Science and Numerical Simulation, vol. 19, no. 10, pp. 3857-3879, 2014.

[25] D. Wang and L. Huang, "Periodicity and global exponential stability of generalized Cohen-Grossberg neural networks with discontinuous activations and mixed delays," Neural Networks, vol. 51, no. 3, pp. 80-95, 2014.

[26] J.-L. Wang, H. Jiang, and C. Hu, "Existence and stability of periodic solutions of discrete-time Cohen-Grossberg neural networks with delays and impulses," Neurocomputing, vol. 142, no. 22, pp. 542-550, 2014.

[27] J. Wang and L. Huang, "Almost periodicity for a class of delayed Cohen-Grossberg neural networks with discontinuous activations," Chaos, Solitons and Fractals, vol. 45, no. 9-10, pp. 1157-1170, 2012.

[28] H. Xiang and J. Cao, "Almost periodic solution of CohenGrossberg neural networks with bounded and unbounded delays," Nonlinear Analysis: Real World Applications, vol. 10, no. 4, pp. 2407-2419, 2009.

[29] Z. Yuan, D. Hu, L. Huang, and G. Dong, "Existence and global exponential stability of periodic solution for Cohen-Grossberg neural networks with delays," Nonlinear Analysis: Real World Applications, vol. 7, no. 4, pp. 572-590, 2006.

[30] F. Zhang, B. Liu, and L. Huang, "Existence and exponential stability of periodic solutions for a class of Cohen-Grossberg neural networks with bounded and unbounded delays," Computers and Mathematics with Applications, vol. 53, no. 9, pp. 1325-1338, 2007.

[31] J.-L. Wang, H.-N. Wu, and L. Guo, "Novel adaptive strategies for synchronization of linearly coupled neural networks with reaction-diffusion terms," IEEE Transactions on Neural Networks and Learning Systems, vol. 25, no. 2, pp. 429-440, 2013.

[32] J.-L. Wang and H.-N. Wu, "Synchronization and adaptive control of an array of linearly coupled reaction-diffusion neural networks with hybrid coupling," IEEE Transactions on Cybernetics, vol. 44, no. 8, pp. 1350-1361, 2014.

[33] Z. Zheng, Theory of Functional Differential Equation, Anhui Education Publishing Press, 1994.

[34] C. Y. Zhang, Pseudo almost periodic functions and their applications [Ph.D. thesis], University of Western Ontario, 1992.

[35] J. Hale, Theory of Functional Differential Equations, Springer, New York, NY, USA, 1977. 


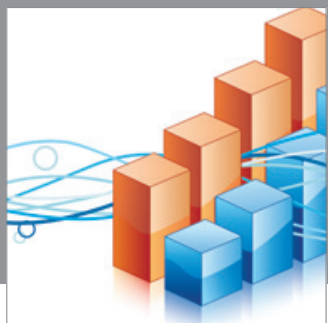

Advances in

Operations Research

mansans

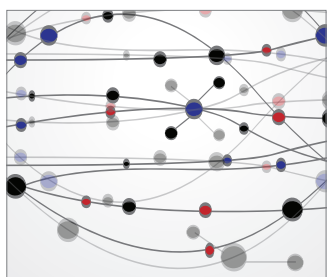

The Scientific World Journal
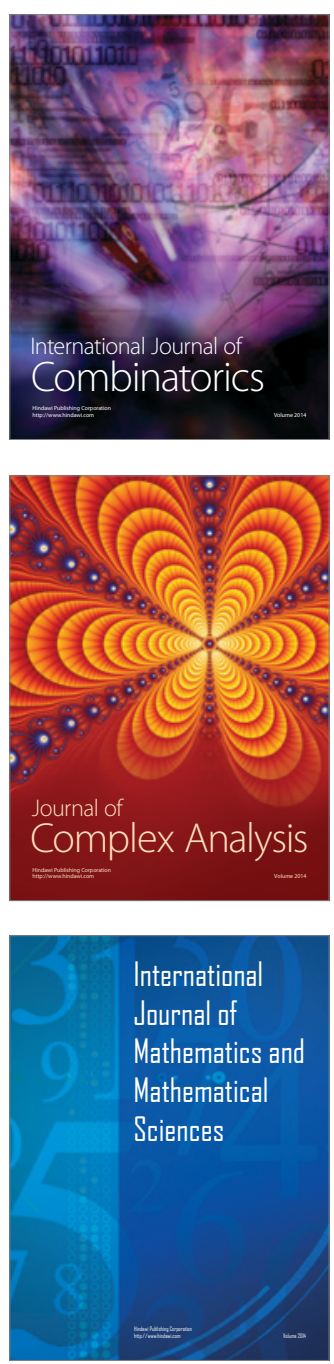
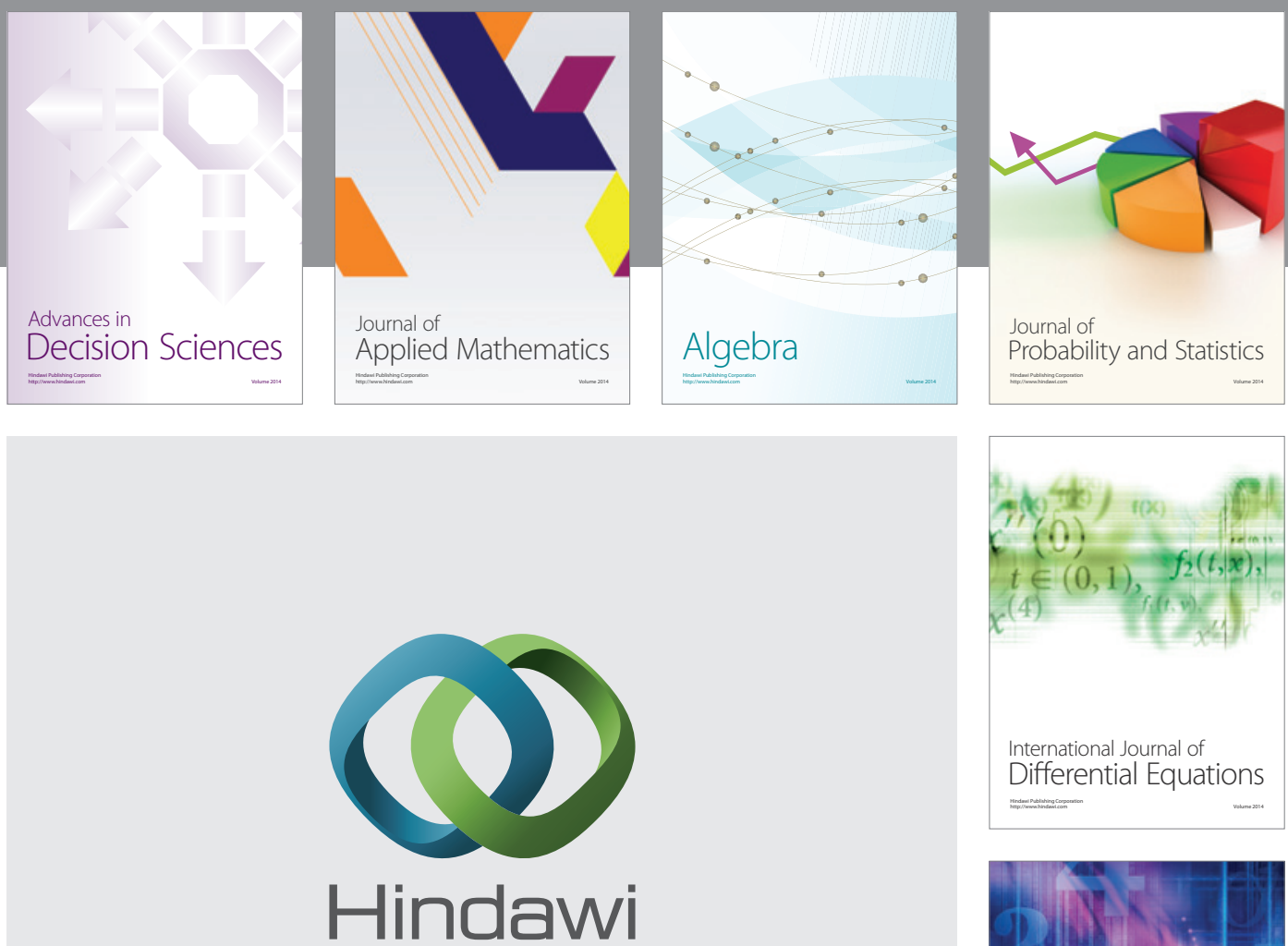

Submit your manuscripts at http://www.hindawi.com
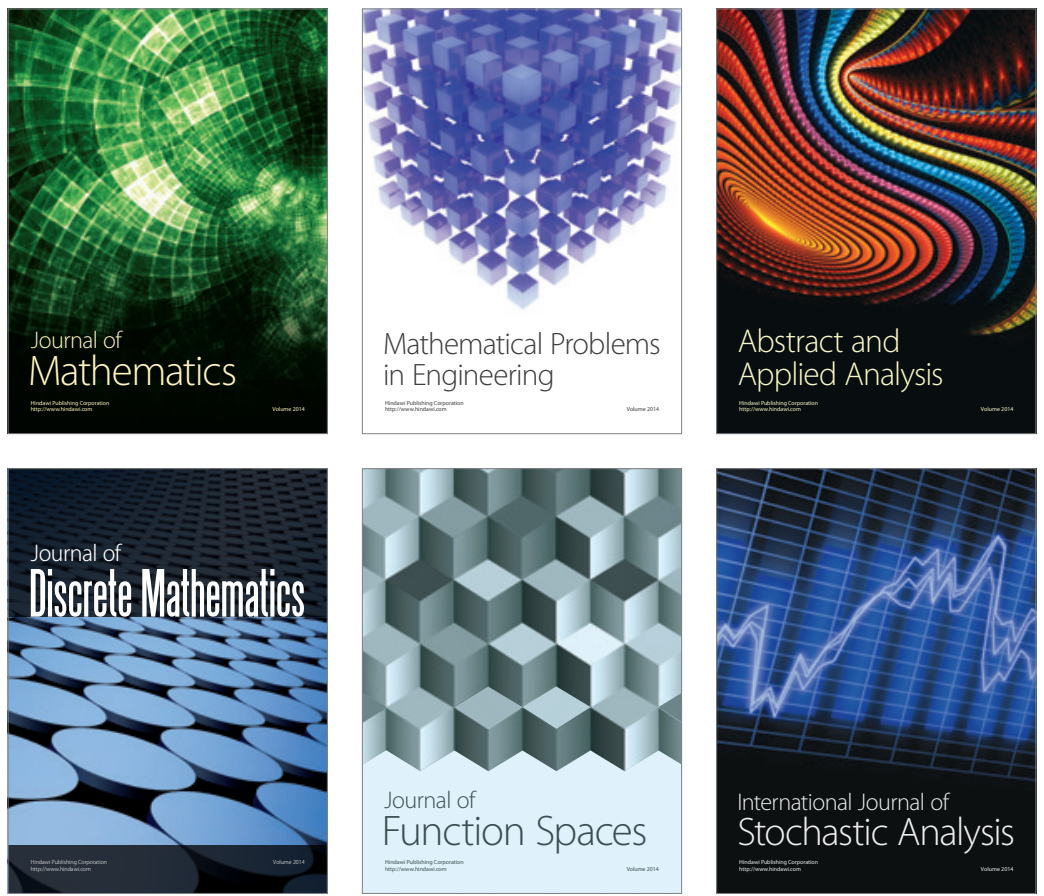

Journal of

Function Spaces

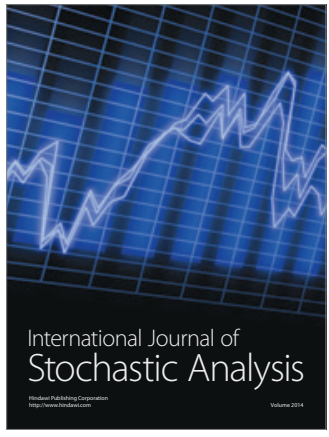

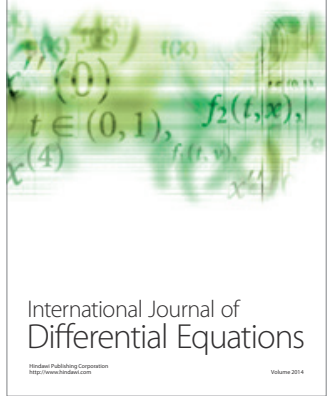
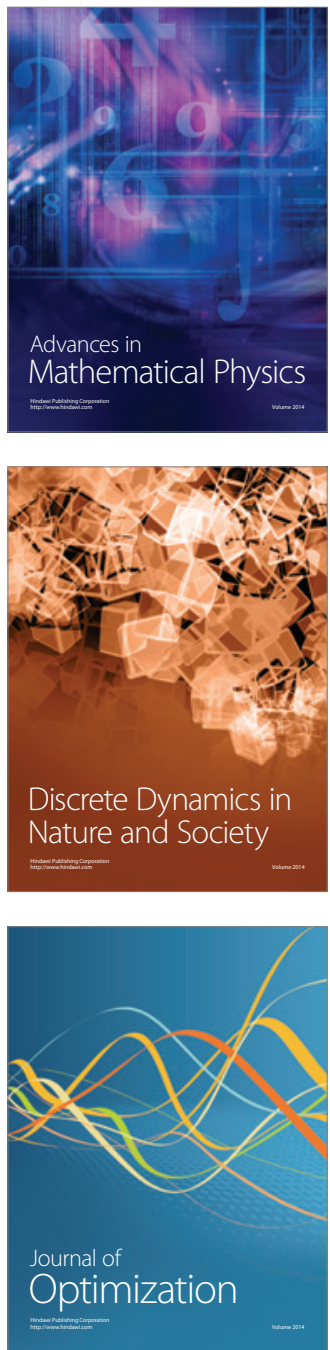